\title{
A trust evaluation scheme of service providers in mobile edge computing
}

\author{
Merrihan Badr Monir Mansour ${ }^{1,2}$, Tamer Abdelkader ${ }^{1}$, Mohammed Hashem AbdelAziz ${ }^{1}$, \\ El-Sayed Mohamed EI-Horbaty ${ }^{1}$ \\ ${ }^{1}$ Faculty of Computer and Information Sciences, Ain Shams University, Cairo, Egypt \\ ${ }^{2}$ Faculty of Business Administration Economics and Political Science, The British University in Egypt, Cairo, Egypt
}

\begin{tabular}{l} 
Article Info \\
\hline Article history: \\
Received Dec 25, 2020 \\
Revised Sep 19, 2021 \\
Accepted Oct 10, 2021
\end{tabular}

Keywords:

Edge computing

Processing performance

Processing throughput

Service level agreement

Service providers

Trust evaluation

\begin{abstract}
Mobile edge computing (MEC) is a new computing paradigm that brings cloud services to the network edge. Despite its great need in terms of computational services in daily life, service users may have several concerns while selecting a suitable service provider to fulfil their computational requirements. Such concerns are: with whom they are dealing with, where will their private data migrate to, service provider processing performance quality. Therefore, this paper presents a trust evaluation scheme that evaluates the processing performance of a service provider in the MEC environment. Processing performance of service providers is evaluated in terms of average processing success rate and processing throughput, thus allocating a service provider in a relevant trust status. Service provider processing incompliance and user termination ratio are also computed during provider's interactions with users. This is in an attempt to help future service users to be acknowledged of service provider's past interactions prior dealing with it. Thus, eliminating the probability of existing compromised service providers and raising the security and success of future interactions between service providers and users. Simulations results show service providers processing performance degree, processing incompliance and user termination ratio. A service provider is allocated to a trust status according to the evaluated processing performance trust degree.
\end{abstract}

This is an open access article under the $\underline{C C B Y-S A}$ license.

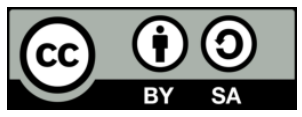

\section{Corresponding Author:}

Merrihan Badr Monir Mansour

Faculty of Computer and Information Sciences, Ain Shams University

El-Khalifa El-Maamoun, Al Obour, Al Qalyubia Governorate, Egypt

Email: merrihan.mansour@bue.edu.eg

\section{INTRODUCTION}

Mobile edge computing (MEC) is a new emerging technology that extends the cloud computing capabilities to the network edge [1], by integrating MEC servers with the mobile network edge [2], through radio access network (RAN) [3], [4]. This permits direct mobile communication between the base network and end users [5], which allows low latency, better quality of service (QoS) [6], high bandwidth access to mobile applications and network information [7]. With the great evolution of mobile devices' capabilities, their owners hold valuable information, apart from the devices' configuration, such as real time knowledge and on-time location awareness of an event. Such mobile capabilities and information are considered great resources in terms of data analysis, processing, and storage media [8]. With the MEC network expansion [9], there is a great increase in service providers offering services. In this context, there could be different service providers offering similar service types, e.g., processing computation and/or storage, were each of them could have different processing performance quality. On the other hand, one service provider could offer 
more than one service type [10]. However, not all services offered by the same service provider could have the same processing performance efficiency. Meanwhile, service users could require different functionalities and have different processing preferences, in terms of cost, storage capacity, processing performance quality and trust degree [11], [12].

Given that, service providers are located in remote locations, most of them are unknown to service users. For this reason, service users hold several doubts such as, dealing with unknown service providers, user's data privacy, service providers' history and processing performance quality. This is mainly due to the lack of previous experience between service providers and users. This creates a level of uncertainty about the fulfilment of service users' various computational needs and expectations, which limits users' dependency on the MEC resources [13]. Many researchers had presented various attempts to build trusted relationships in edge computing paradigms [14]. This is to provide an efficient trust evaluation scheme for the available services provided by service providers, to secure future users' interactions [15], [16]. In mobile edge computing, a secure multi-tier model was proposed in [17]. In this protocol, it was assumed that the higher degree of trust, the less security measures could be taken by a node and vice versa. But unfortunately, it did not consider sudden attacks occurring for a trusted node, such as hacking. A trust evaluation scheme was presented in [18], were it computes service providers' identity, hardware capabilities and behavioral trust in the MEC network. The main limitation of this scheme is that it mainly depends on users' feedback opinion to compute trust.

An integrated trust evaluation model was depicted in [19], to evaluate service providers' identity, historical behavior and quality of service offered. Trust computation was time consuming in this model, due to the complexity of the equations. In [20], a trust assessment protocol was developed that monitors and analyze traffic flow of interactions between service users and providers to evaluate trust. However, no performance parameters were evaluated. Another attempt to evaluate service providers' performance during their interactions is the issuance of a service level agreement (SLA) [21]. An SLA is an agreed upon document between a service provider and user, that specifies the required task description and application requirements [22]. Yet, this is not sufficient to secure service users, since not all service providers abide to the SLA statements thoroughly. However, there is a lack of a standard SLA format. As shown, each of the previously mentioned protocols measured cloud services using different parameters. There is not a unified scheme that could evaluate service providers processing performance. Meanwhile, node history was not captured, which gives a chance for an entity to behave maliciously, knowing that it would not be recognized in the future. This increases service users' fears and prevents them from relying on the cloud and edge computing paradigms to fulfil their computational needs.

This paper presents a unified trustworthy evaluation model that evaluates service providers' processing performance, to distinguish trustworthy providers. The main contributions of this paper are: i) service providers' processing performance evaluation in terms of processing success ratio and throughput, ii) processing incompliance and user termination ratio computation of service providers, iii) development of a penalty system to track malicious actions committed by service providers, and iv) assignment of service providers to relative trust status. This would help service users in their service providers' selection and optimizes the security of future interactions, which enhances the MEC network expansion [23].

The rest of this paper is organized as follows; section 2, introduces the proposed architecture method, functional algorithms, and their description. Section 3 shows the results and discussion. Finally, the conclusion and future work are presented in section 4.

\section{PROPOSED ARCHITECTURE METHOD}

The proposed architecture evaluates the processing performance of service providers in the MEC network. In this model, a service provider is evaluated according to its processing performance quality and not by the quantity of hardware or software resources that it possesses, e.g., storage space, number of processors and RAM. The main protocol entities, proposed functions, equations, and algorithms are described in subsection 2.1 to 2.6 .

\subsection{Main protocol entities and their equivalent tasks}

The main acting entities in the proposed scheme are service provider SP, service user SU, cloud broker (CB), network provider (NP) and cloud service manager (CSM) [24]. The relationship between the protocol entities is shown in Figure 1 and detailed below.

- Service provider $\mathrm{SP}_{\mathrm{i}}$ : [25] a service provider "i", i $\in \mathrm{I}$, where $\mathrm{I}$ is the set of service providers. A service provider may be a small entity offering one service of one type, or a big organization that owns several hardware and software resources and offers several services of different job types. In case a service 
provider $\mathrm{SP}_{\mathrm{i}}$ provides more than one service type, it is referred to as $\mathrm{Sr}_{\mathrm{i}}$. For simplicity, we will be referring to each service provider as $\mathrm{Sr}_{\mathrm{i}}$, throughout this paper.

- Service user $\mathrm{SU}_{\mathrm{j}}$ : a service user " $\mathrm{j}$ ”, $\mathrm{j} \in \mathrm{J}$, where $\mathrm{J}$ is the set of service users. A service user could be an entity or organization that requests a specific service to be performed over the network and pays for it [26].

- Cloud broker $\mathrm{CB}_{\mathrm{u}}$ : a cloud broker " $\mathrm{u}$ ", u $\in \mathrm{U}$, where $\mathrm{U}$ is the set of cloud brokers. CB is an entity that mainly helps service users to find appropriate service providers to fulfil their computational needs, and it is being paid for this job. CB works as a local entity per area, where it should be aware of all available service providers and their offered service type. $\mathrm{CB}$ could communicate with other entities outside its area to reach suitable service providers [22]. CB is considered as a semi trusted entity that is not allowed to reveal service provider or service user private information, such as own opinion, as it could have a personal benefit. It also acts as a transmission and storage medium between service provider, user and CSM for certain encrypted information as shown below.

- Cloud service manager (CSM): is considered a fully trusted authorized entity that is responsible for registering cloud brokers, service providers and service users to the MEC network through a network provider. CSM evaluates the processing performance and trust status of service providers [27]. It also checks the status and validity of cloud brokers periodically to ensure secure communication medium between service providers and users. Therefore, CSM should maintain high computational capabilities and covers a wide geographical region.

- Network provider $\mathrm{NP}_{\mathrm{w}}$ : a network provider "w", w $€ \mathrm{~W}$, where $\mathrm{W}$ is the set of network providers. NP is responsible for communication, data transmission and network efficiency, between all the above entities. Note that, there could be more than one network provider located per geographic area [12].

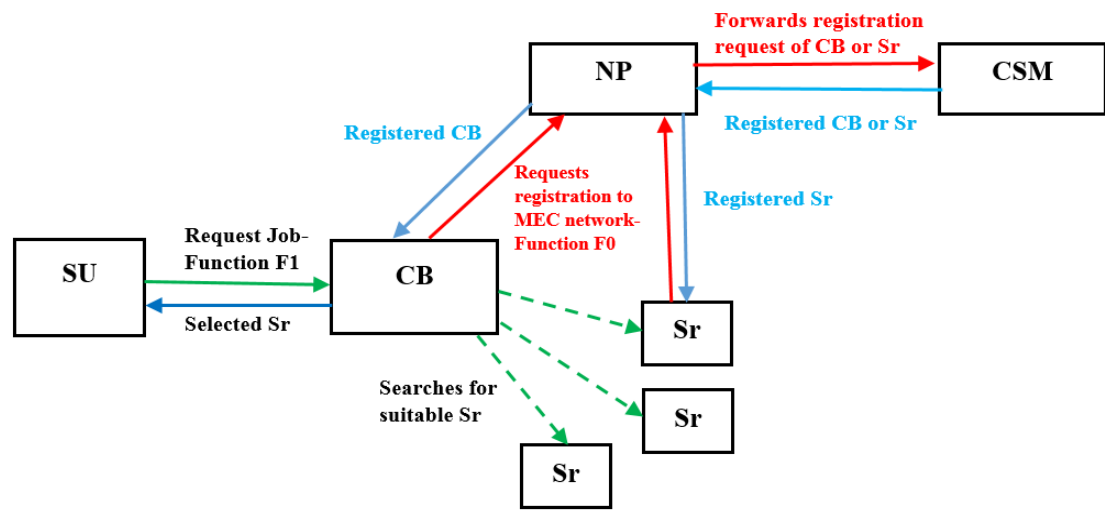

Figure 1. Main protocol entities relationship

However, a cloud broker, service provider or service user could deal with more than one network provider [28]. While a service provider could accept jobs from more than one cloud broker, a service user can also deal with more than one cloud broker to request different computational tasks. All of the above entities are authenticated in the MEC network by their unique identity, which is out of the scope of this paper.

\subsection{List of assumptions}

The proposed scheme considers the following assumptions:

a) There are three job types requested over the MEC network; type 1: storage request (storing massive terabytes, e.g., videos), type 2: computational processing request (jobs that require high processing speed/capabilities), type 3: requesting both of them. These jobs are the most commonly requested processes in the cloud computing paradigm and MEC network;

b) If the same service provider $S P_{i}$ offers more than one job type, known as $S r_{i}$, were $\mathrm{r} \in$ type 1 , type 2 , type 3$\}$. This does not mean that SP has the same computational efficiency for all job types. For instance, it could be powerful in one job type, e.g., storage, and weak in another, e.g., processing efficiency or vice versa;

c) The same service provider $S P_{i}$ gives equal usage and benefits of its hardware and software resources to all its services and users;

d) Job processing, storage and execution isolation is considered as a default action by a service provider [29]; 
e) Since each service user could have different priorities and intentions, the proposed scheme asks the service user for a priority list of preferences and responds with a recommendation list accordingly;

f) Batch processing is assumed for trust computation. All processes of the same job type per service provider $S r_{i}$ are gathered per computational interval (e.g., month);

g) Trust evaluation is performed per process accepted by $S r_{i}$;8) A network provider of each of $S r_{i}, S U_{j}$ and $C B_{u}$, have a limited contribution in the proposed scheme as discussed below.

\subsection{Processing performance computational equations}

The proposed trust scheme measures the processing performance $\mathrm{P}\left(S r_{i}\right)$ of service provider $S P_{i}$ which could own more than one service $S r_{i}$. However, jobs of the same type, are evaluated per service provider $S r_{i}$, as mentioned previously. Noting that, $\mathrm{P}$ consists of different weighted parameters and computed by the CSM, as described below.

Let each requested job of any type be known as process "a", a $€ \mathrm{~A}$, where $\mathrm{A}$ is the total number of processes executed at $S r_{i}$, but distinguished by their job type. Each process "a" has a separate service level agreement, even if it is performed by the same $S P_{i} / S r_{i}$. Assume the components of an SLA of process "a" executed by $S r_{i}$ be processing cost $\left(S C_{i a}\right)$, storage capacity GB/TB $\left(S S_{i a}\right)$, duration of maintenance hr/min $\left(S M_{i a}\right)$, and agreed estimated execution time $\mathrm{hr} / \mathrm{min}\left(S E_{i a}\right)$ [30]. All SLA's components are rated by $S U_{j}$ after the job is ended or terminated [31]. The proposed scheme constitutes of eight equations, as detailed below.

Let $T_{v}$ be actual processing execution time of process "a" at $S r_{i}$, where "a" start time is $P a_{s t}$ and end time is $P a_{e t}$. Hence,

$$
T_{v}=P a_{e t}-P a_{s t}
$$

Assume the time difference between the estimated agreed time $S E_{i a}$ and actual processing execution time $T_{v}$, be $T R_{i a}$ (Time compliance), therefore,

$$
\begin{aligned}
& T R_{i a}=S E_{i a}-T_{v} \\
& T R_{i a}=\left[\begin{array}{ll}
\geq 0 & " a " \text { completed within } \mathrm{SE}_{\mathrm{ia}} \\
<0 & \mathrm{SE}_{\mathrm{ia}} \text { time incompliance }
\end{array}\right]
\end{aligned}
$$

Given that each process " $\mathrm{a}$ " should end in one of the following four states $\left\{\mathrm{P}_{\mathrm{E} 1}, \mathrm{P}_{\mathrm{E} 2}, \mathrm{P}_{\mathrm{T} 1}, \mathrm{P}_{\mathrm{T} 2}\right\}$ :

$$
\begin{aligned}
& {\left[\begin{array}{lll}
\mathrm{P}_{\mathrm{E} 1} & \text { Process ended by } \mathrm{Sr}_{\mathrm{i}} & \text { complete } \\
\mathrm{P}_{\mathrm{E} 2} & \text { Process ended by } \mathrm{Sr}_{\mathrm{i}} & \text { incomplete }
\end{array}\right]} \\
& {\left[\begin{array}{lll}
\mathrm{P}_{\mathrm{T} 1} & \text { Process terminated by } \mathrm{SU}_{\mathrm{j}} & \mathrm{T}_{\mathrm{V}}>\mathrm{SE}_{\mathrm{ia}} \\
\mathrm{P}_{\mathrm{T} 2} & \text { Process terminated by } \mathrm{SU}_{\mathrm{j}} & \mathrm{T}_{\mathrm{v}}<\mathrm{SE}_{\mathrm{ia}}
\end{array}\right]}
\end{aligned}
$$

Let $P_{E 1-} T, P_{E 2 \_} T, P_{T 1-} T$ and $P_{T 2-} T$ be the total number of $P_{E 1}, P_{E 2}, P_{T 1}$ and $P_{T 2}$ respectively. Let the rated SLA, be $S L A_{i a_{-}} R$, and the total number of $S L A_{i a_{-}} R$ implies the total number of ended jobs/processes, "A". Therefore, the average processing success rate $P_{A V}\left(S r_{i}\right)$ can be measured by,

$$
P_{A V}\left(S r_{i}\right)=\frac{P_{E 1 \_} T}{A}
$$

On the other hand, processing incompliance $\operatorname{PI}\left(S r_{i}\right)$ or failure ratio, can be calculated as,

$$
P I\left(S r_{i}\right)=\frac{P_{E 2 \_} T+P_{T 1-} T}{A}
$$

The user termination ratio $U T R\left(S r_{i}\right)$ can be measured by,

$$
\operatorname{UTR}\left(S r_{i}\right)=\frac{P_{T 2-} T}{A}
$$

In case $\operatorname{UTR}\left(S r_{i}\right)$ exceeds a certain threshold, a warning is issued to alarm the relevant service provider of its high user termination ratio. The processing throughput $\operatorname{PT}\left(\boldsymbol{S} \boldsymbol{r}_{\boldsymbol{i}}\right)$ will be measured by 
considering the number of completed successful processes $P_{E 1}$, per computational interval and given certain points for each range of values by,

$$
\operatorname{PT}\left(S r_{i}\right)=\operatorname{Points}\left(S r_{i}\right)
$$

for $1 \leq \mathrm{P}_{\mathrm{E} 1 \_\mathrm{T}} \quad / /$ Function $\mathrm{F} 3$.

After all, the processing performance of a service provider $\mathrm{P}\left(\boldsymbol{S} \boldsymbol{r}_{\boldsymbol{i}}\right)$ will be computed as,

$$
\mathrm{P}\left(S r_{i}\right)=\frac{P_{A V}\left(S r_{i}\right)+\mathrm{PT}\left(S r_{i}\right)}{2}
$$

Noting that the total number of processes executed, "A", at service provider $S r_{i}$ of $S P_{i}$, per computational interval, equals;

$$
A=P_{E 1 \_} T+P_{E 2 \_} \mathrm{T}+P_{T 1 \_} T+P_{T 2 \_} T
$$

as process "a", should end in one of these four states. The processing capacity $P C_{i}$ of a service provider $S r_{i}$, is a predetermined value by the service provider specified during the registration process. Each of the above equations is applied in the below functional algorithms, as part of the trust computation process.

\subsection{Functional algorithms and description}

The proposed protocol is composed of eleven functional algorithms, which leads to measuring the processing performance of a service provider $S r_{i}$ and assigning it to a trust status. It also measures the processing incompliance and user termination ratio per service provider. A penalty system is also presented to identify any malicious action performed by the participating entities. Table 1 shows each function name and aim, the consequent action performed with the relevant algorithm figure number. Figure 2 presents the protocol architecture and the relationship between the eleven functions. Dashed lines indicate that this function may or may not be called.

Table 1. Proposed scheme functions

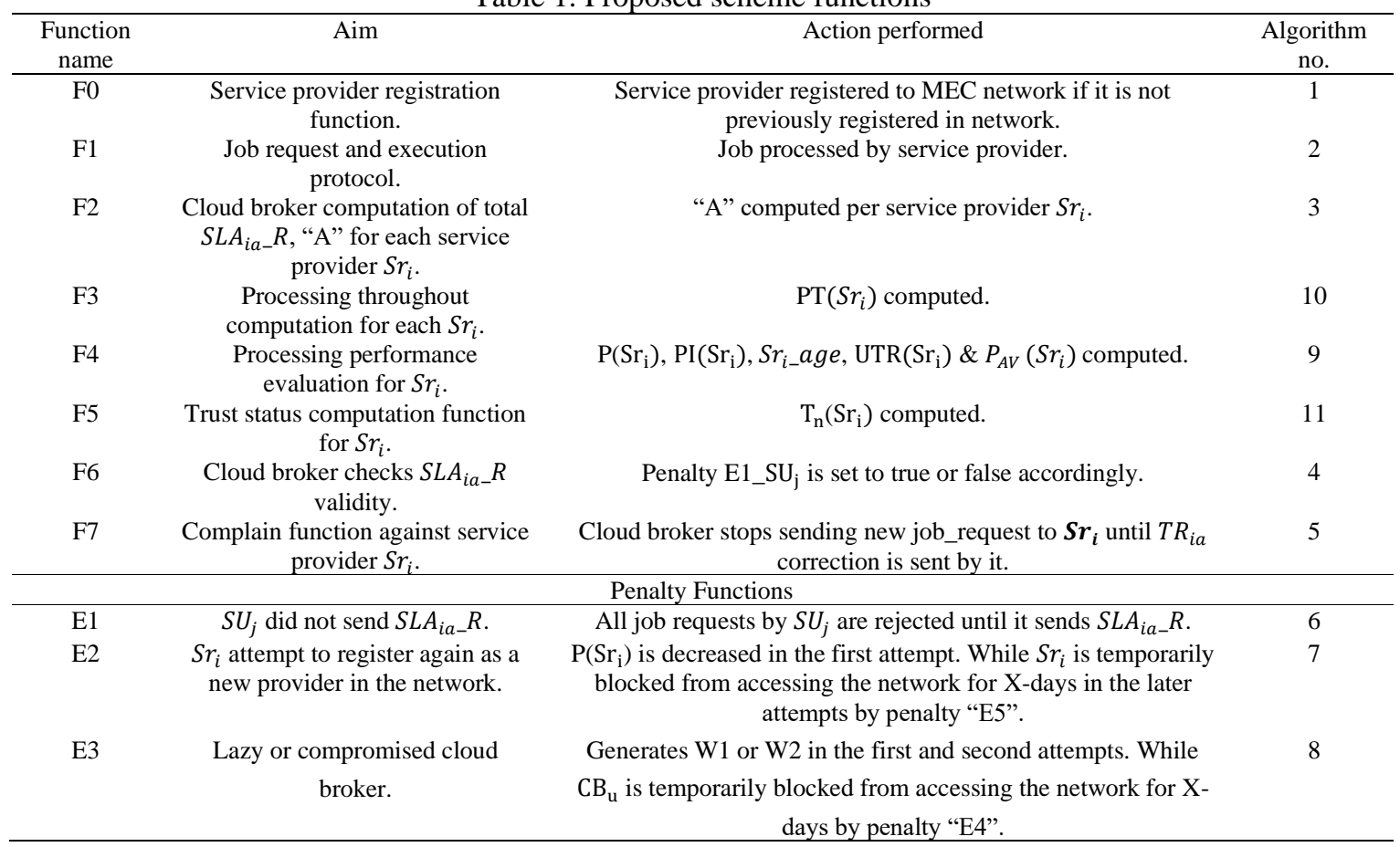

\subsection{Proposed penalty protocol}

The penalty protocol is presented as part of the proposed architecture. It mainly aims to track service providers malicious actions, which helps in their processing performance and trust status computation process. However, the penalty protocol achieves the following: i) it shows the type of wrong action 
performed by the involved entity; ii) it encounters several malicious action types expected to happen, such as an existing service provider attempt to register as a new one, in order to hide its bad history; iii) it counts the number and type of malicious actions performed per computational interval; and iv) imposes a penalty according to each malicious action type performed by the accused entity.

The penalty scheme also monitors cloud brokers and service users' actions in a limited manner, since this is out of the scope of this paper. This provides more secure transactions between service providers, users, and cloud brokers in the MEC environment. Table 2 states each penalty name, its description, and consequences. All of the above functions are described in the following subsections with their relevant algorithms.

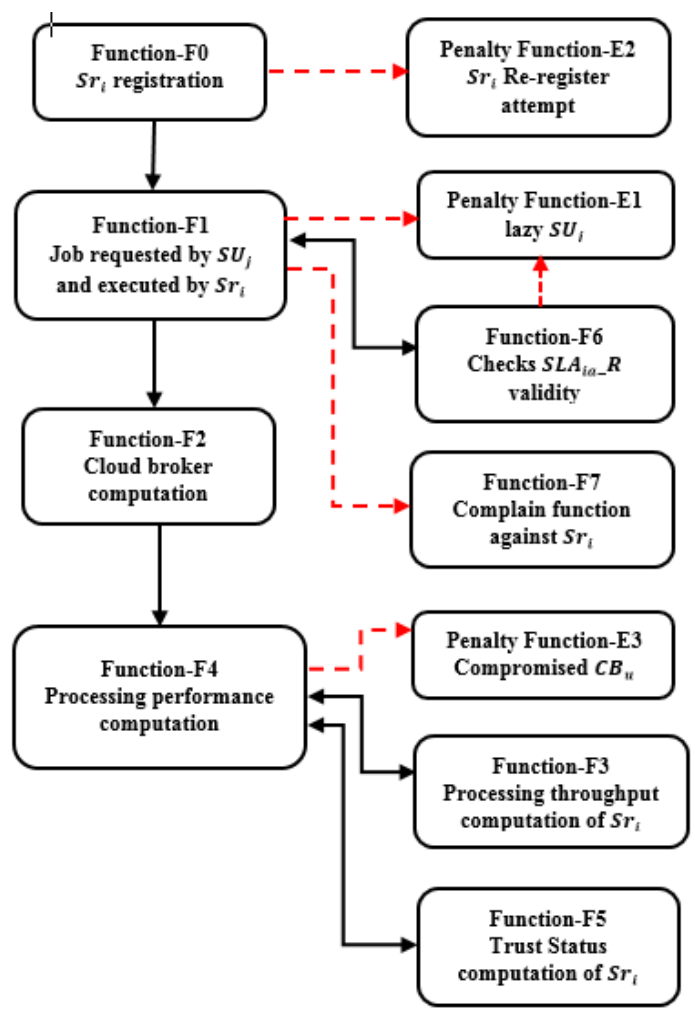

Figure 2. Functions relationship diagram

Table 2. Penalties and their consequences

\begin{tabular}{|c|c|c|}
\hline Penalty name & Description & Consequences \\
\hline E1_SU & $\begin{array}{c}S U_{i} \text { did not send to } C B_{u} \operatorname{rated}\left(S L A_{i a_{-}} R\right) \text { after process } \\
\text { ended/terminated. }\end{array}$ & $\begin{array}{l}\text { Job request rejected until sending the pending } \\
\qquad S L A_{i a_{-}} R \text {. }\end{array}$ \\
\hline $\mathrm{E} 2 \_S r_{i}$ & $S r_{i}$ first attempt to register again in the MEC network. & Request rejected, $P\left(S r_{i}\right)$ decreased. \\
\hline E4_CB & $\begin{array}{l}\text { Third attempt of cloud broker to delay its computational job, } \\
\text { after being warned by "E3". Compromised } \mathrm{CB}_{\mathrm{u}} \text {. }\end{array}$ & $\begin{array}{l}\text { Cloud broker is temporarily blocked from the } \\
\text { network by CSM. }\end{array}$ \\
\hline
\end{tabular}

\subsection{Functional algorithms}

In this section, each of the above mentioned functions in Table 1, are described below with their relative algorithms. The penalty functional algorithms are also detailed below, together with their generated warnings, and actions performed accordingly.

\subsubsection{Service provider registration}

A service provider $S P_{i}$ could own more than one service, thus service registration to the MEC network is performed per service $S r_{i}$ \{job type 1,2, or 3$\}$ of service provider $S P_{i}$, with the aid of a network 
provider. Algorithm1, shows the service provider registration algorithm 1, function F0, and its flowchart in Figure 3.

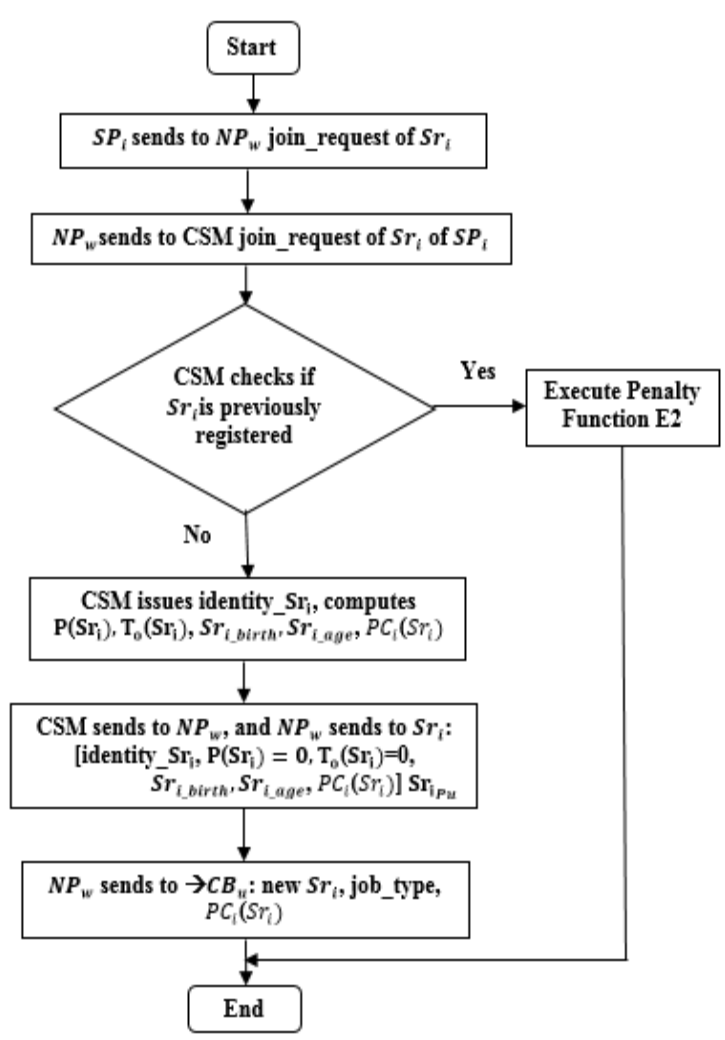

Figure 3. Service provider registration flowchart

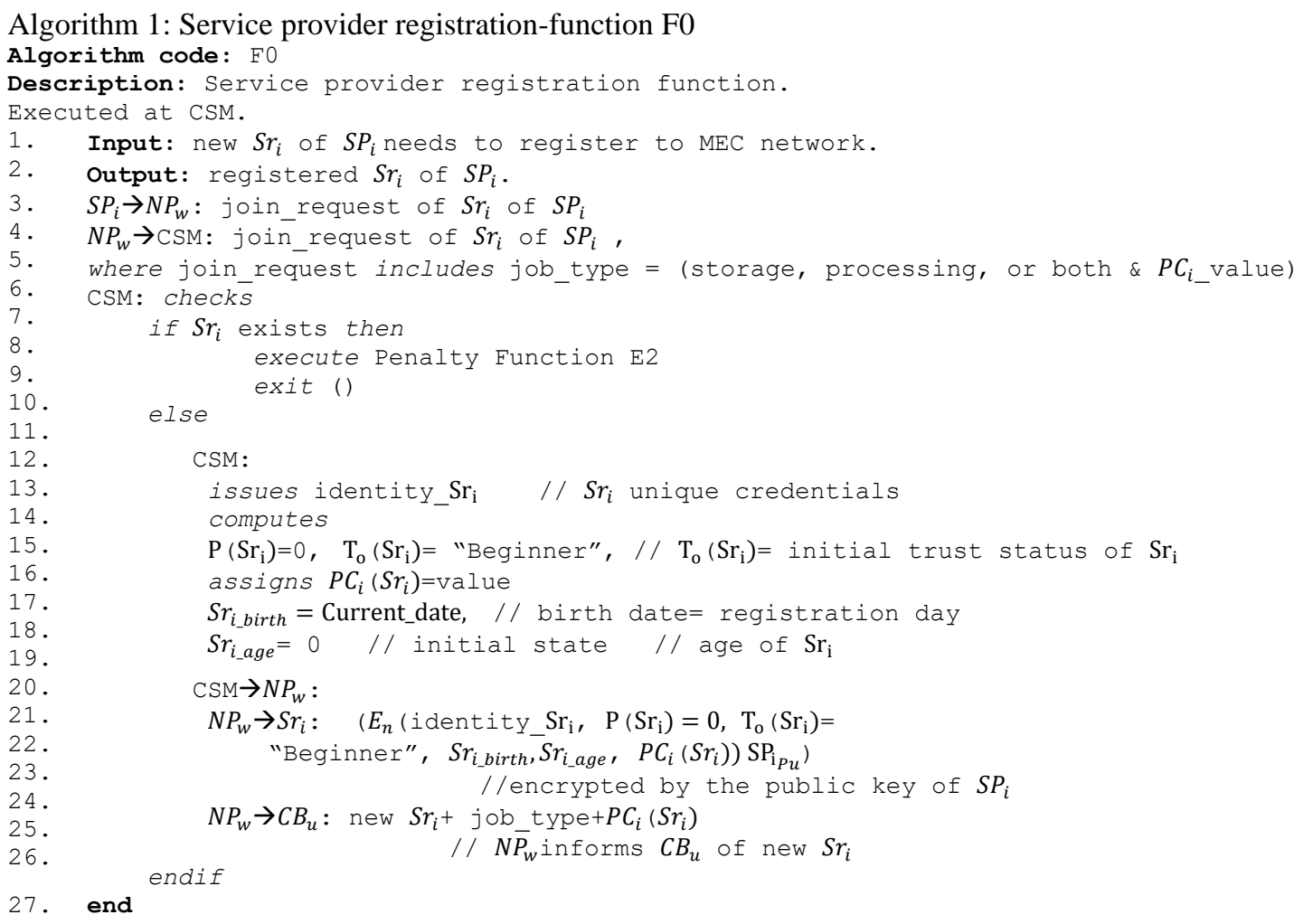


By the completion of function F0, a new service $S r_{i}$ of service provider $S P_{i}$, is now registered to the MEC network, with a unique identity number to be authenticated and distinguished among other service providers. Service provider's unique identity is issued and saved by the CSM. On the other hand, a network provider should have a list of all available registered service providers and must exchange it with other network providers, if any exists, within the same region. A network provider also must continuously update cloud brokers with the newly registered or deactivated service providers. Cloud brokers by return, will be aware and updated with service providers' status in their own area, which enhances service provider selection process by users. This minimizes the communication overhead between the participating entities in the MEC network.

\subsubsection{Job request scenario algorithm}

Algorithm 2, shows the job request scenario algorithm steps. A service user is expected to request one of the previously mentioned job types from a cloud broker, which in return searches for an appropriate service provider. If $S U_{j}$ did not send rated SLA for its previously ended job in the MEC network, this user is penalized by being prohibited from requesting further jobs through function E1, described in section 2.6.4, until it sends the required rated SLA. Otherwise, $C B_{u}$ asks $S U_{j}$ to choose from a priority list its preferences, as shown in algorithm 2. Upon $S U_{j}$ feedback, $C B_{u}$ sends a recommendation list of available service providers, with respect to the chosen priorities [32]. $S U_{j}$ chooses a service provider and informs the $C B_{u}$ of its choice.

Consequently, $C B_{u}$ checks the chosen service provider processing capacity $P C_{i}\left(S r_{i}\right)$ limit. If $P C_{i}\left(S r_{i}\right)$ is not maximum, the cloud broker starts direct communication between the service provider and user. Given that, each job will have a separate SLA, chosen $S r_{i}$ sends an initial SLA, $S L A_{i a_{-}} I$, to $S U_{j}$ for approval. Upon SLA approval by both parties, requested job processing starts, were $S r_{i}$ informs $S U_{j}$ of the job start time; $P a_{s t}$. Job processing is ended or terminated in one of the previously mentioned four states, $\left(P_{E 1}, P_{E 2}, P_{T 1}, P_{T 2}\right)$ by either the $S r_{i}$ or $S U_{j}$. Consequent steps take place accordingly as stated in Figure 2 . In all cases, $S U_{j}$ should send rated $S L A_{i a-} R$.

While $\mathrm{TR}_{\mathrm{ia}}$ (time compliance, equation 2), is computed by $S r_{i}$, it should be approved by $S U_{j}$ within a time threshold, to avoid holding an opened transaction for a long time intentionally by any entity. In case, $S U_{j}$ requests $\mathrm{TR}_{\mathrm{ia}}$ correction, $S r_{i}$ should reply with the corrected $\mathrm{TR}_{\mathrm{ia}}$ within a time threshold, otherwise complain function F7 (detailed in section 2.6.3) is called. Upon $\mathrm{TR}_{\mathrm{ia}}$, agreement, function F6 (algorithm 4) is called to check the validity of rated $S L A_{i a_{-}} R$ (depicted in section 2.6.3). The rated $S L A_{i a_{-}} R$ is encrypted by the public key of the CSM, which will handle trust computation process. This is performed using asymmetric encryption techniques, such as public key infrastructure (PKI) [33]. Using PKI, ensures secure trust computation, information integrity and confidentiality, while securing future user interactions [34].

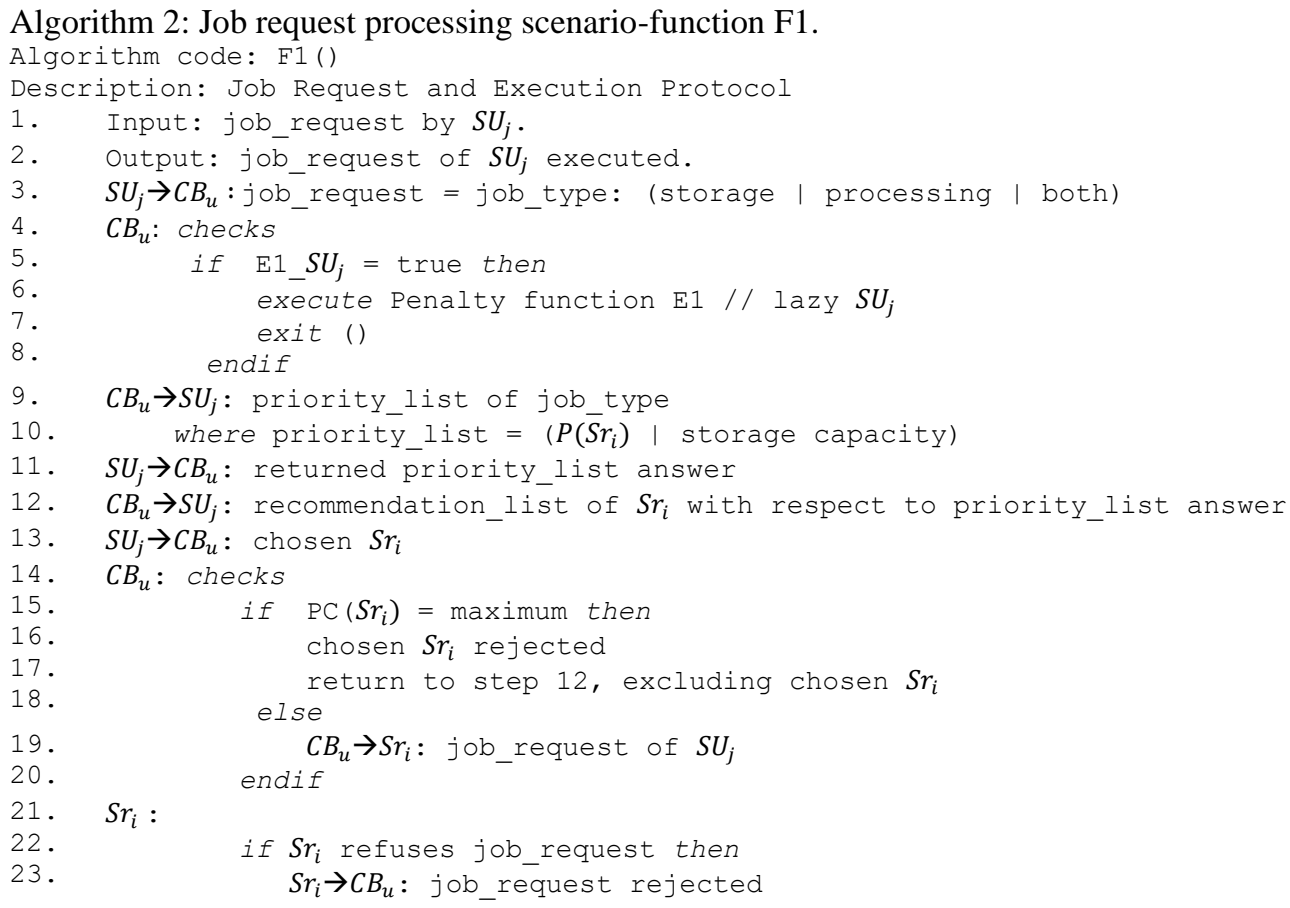




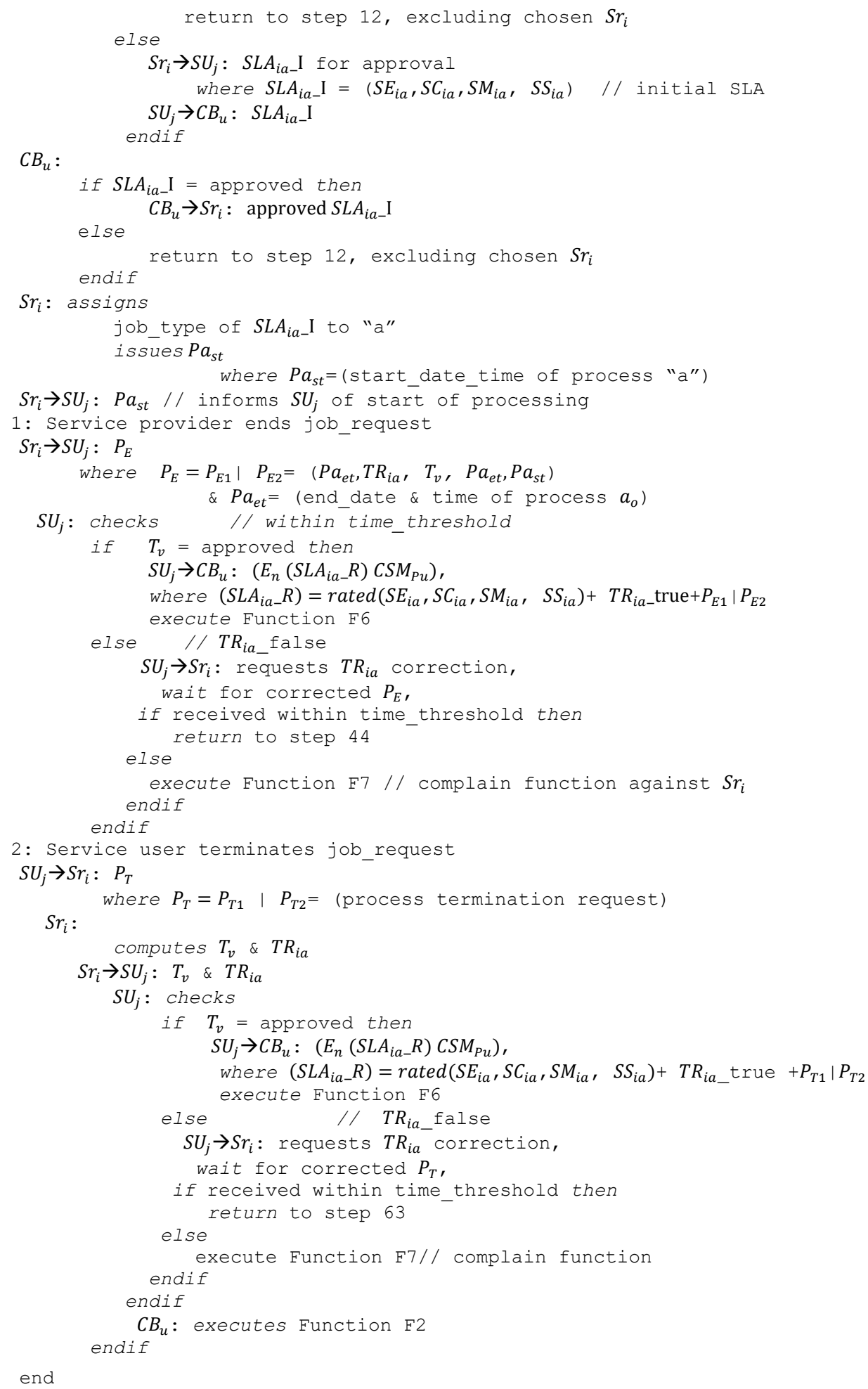

Upon the completion of function F1, $C B_{u}$ gathers all rated SLAs of $S r_{i}$, per computational interval and starts its computation process as described below.

\subsubsection{Cloud broker computational algorithms}

Functions (F2, F6 and F7) are executed by the cloud broker, as explained below. In Function F2 presented in algorithm $3, C B_{u}$ is responsible to collect all the rated $S L A_{i a_{-}} R$ per $S r_{i}$, counts and sends them as a batch of rated SLA's $\left(S L A_{i a_{-}} R n\right)$ to the CSM periodically for trust computation. This batch is sent encrypted by the public key of the CSM and stamped with the date of F2 function execution " $Y d_{i a}$ ". 
Algorithm 3: Cloud broker computational-function F2

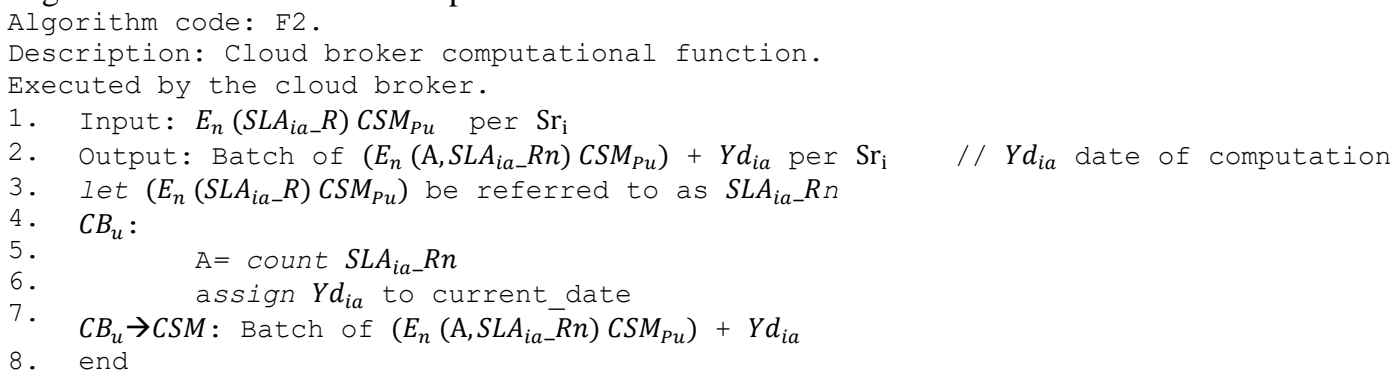

Algorithm 4 of Function F6, is called by function F1, where $C B_{u}$ checks if $S U_{j}$ had sent rated SLA of its last transaction. If not, then $C B_{u}$ executes penalty function E1 to penalize $S U_{j}$, for its laziness. Function F7 presented in algorithm 5, is called by function F1, where a $C B_{u}$ checks whether $S r_{i}$ had sent corrected $T R_{i a}$ or not, within a time_threshold. If not sent, $C B_{u}$ broadcasts message $M_{T R}$ accordingly to all network providers, in order to stop dealing with the relevant $S r_{i}$ until sending corrected $T R_{i a}$. The network provider broadcasts $M_{T R}$ to other cloud brokers as stated in algorithm 5.

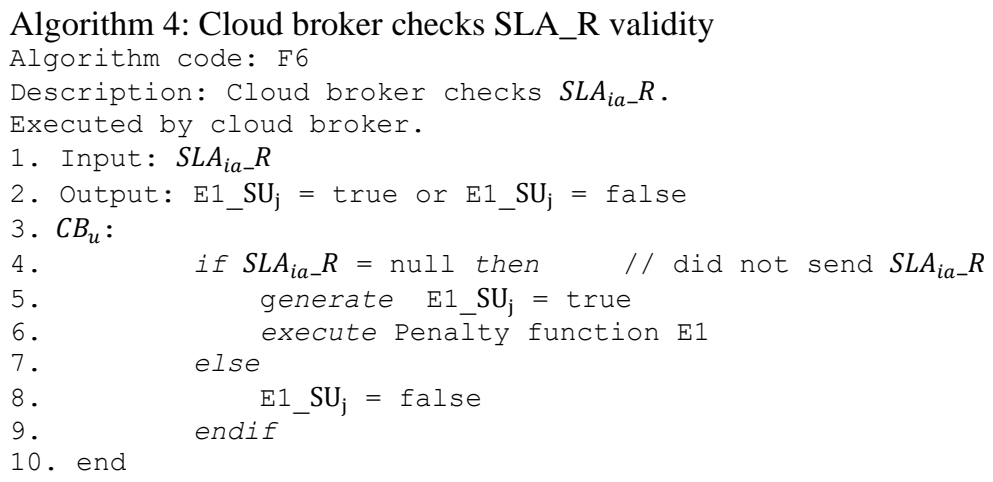

Algorithm 5: Cloud broker complain function against service provider

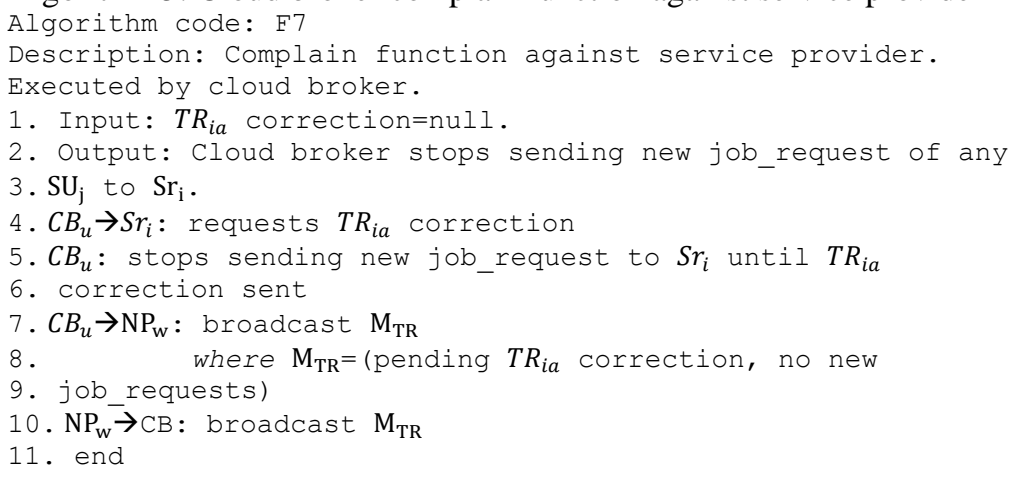

\subsubsection{Penalties computational algorithms}

Three penalty functions (E1, E2, E3) introduced in algorithms 6, 7 and 8 respectively, are described below. Algorithm 6 describes penalty function E1, which is called by function F6, in case $S U_{j}$ did not send rated $S L A_{i a_{-}} R$. In this function, a network provider broadcasts warning message $M_{S L A}$ to all cloud brokers, to warn them not to accept any job requests from $S U_{j}$ until sending the rated $S L A_{i a_{-}} R$ as shown in algorithm 6.

Algorithm 6: Penalty function E1

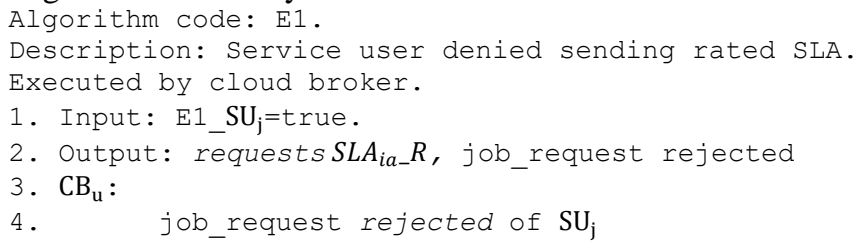


5. $\quad \mathrm{CB}_{\mathrm{u}} \rightarrow \mathrm{NP}_{\mathrm{w}}\left(\mathrm{SU}_{\mathrm{j}}\right): \mathrm{M}_{\mathrm{SLA}}$

6. where $\mathrm{M}_{\mathrm{SLA}}=$ (pending $S L A_{i a_{-}} R$, job request rejected)

7. $\mathrm{NP}_{\mathrm{w}}\left(\mathrm{SU}_{\mathrm{j}}\right) \rightarrow \mathrm{CB}$ : broadcasts $\mathrm{M}_{\mathrm{SLA}}^{-}$to nearby $\mathrm{CB}_{\mathrm{u}}, \forall \mathrm{u}$,

9. end

$\mathrm{CB} \rightarrow \mathrm{SU}_{\mathrm{j}}: \mathrm{E}_{1} \mathrm{SU}_{\mathrm{j}}$, requests $S L A_{i a-} R$

Algorithm 7 presents penalty function E2, were it's called by function F0, in case CSM discovers that a previously registered $S r_{i}$, is trying to register as a new provider to the MEC network. Hence, CSM imposes penalty E2, which decreases $S r_{i}$ processing performance value, in the first attempt. In case this action is repeated again, malicious $S r_{i}$ is temporarily blocked from accessing the MEC network by penalty E5. Consequently, CSM sends warning message $M_{y}$ to the involved $C B_{u}$ and requests from it to find a replacement service provider, to delegate malicious $S r_{i}$ tasks to a newly selected provider. $C B_{u}$ broadcasts this message to other cloud brokers and service providers, in order not to deal with malicious service provider temporarily.

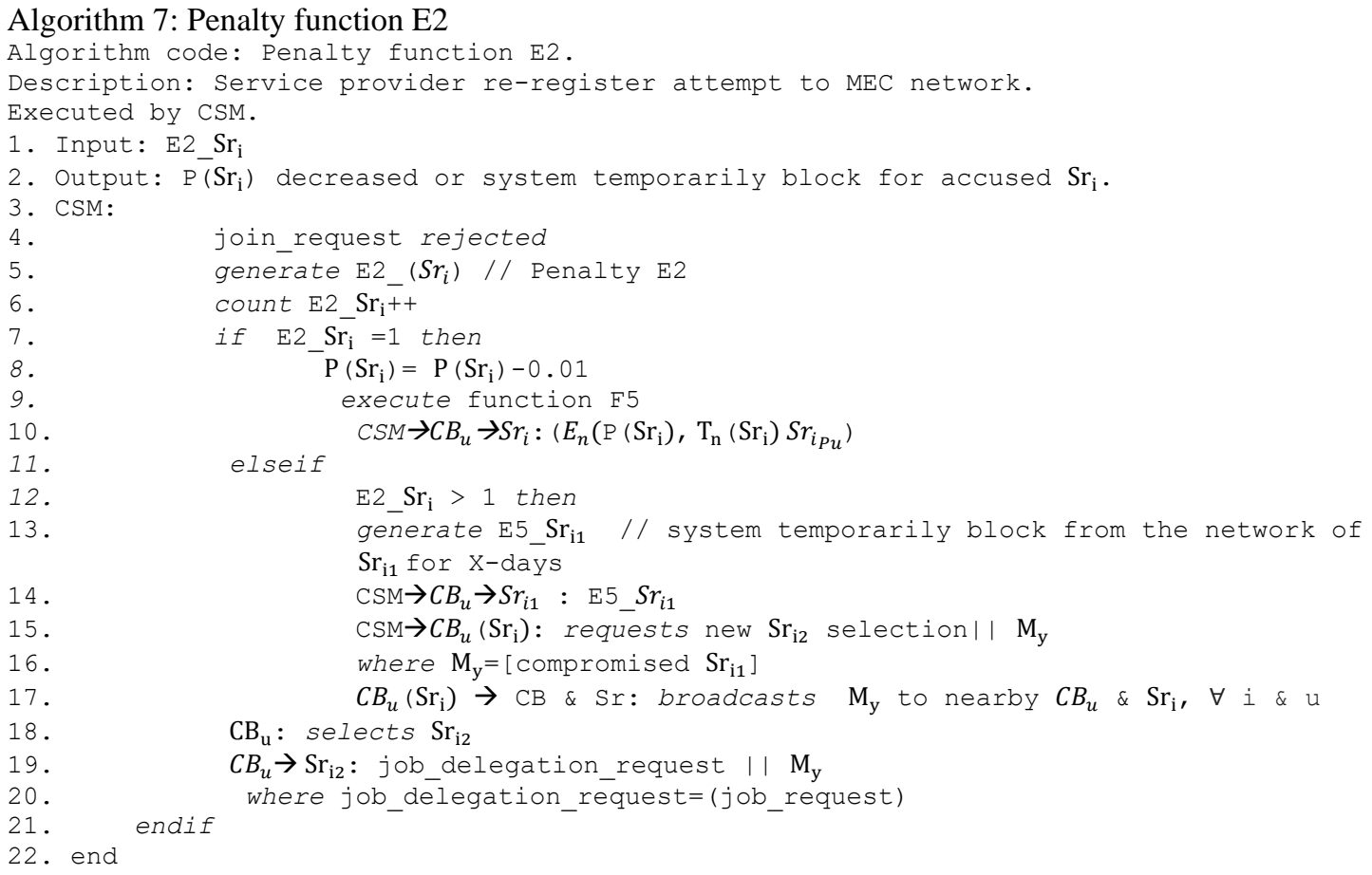

On the other hand, penalty function E3, shown in algorithm 8, is called by function $\mathrm{F} 4$, in two cases; case1: a $C B_{u}$ postpones sending the total number $S L A_{i a_{-}} R$, "A", per $S r_{i}$; case2: $C B_{u}$ adjusts its computation time $\left(Y d_{i a}\right)$ as a malicious action, during its processing of function $\mathrm{F} 2$. In both cases, the $C B_{u}$ is claimed to be accused. $C B_{u}$ is warned by warning number "W1", and a message is sent to it by the CSM. If one of these actions is repeated again, CSM sends warning number "W2" to the accused $C B_{u}$. If the $C B_{u}$ performs any of these malicious actions for the third time, then penalty E4 is imposed by CSM, which deactivates $C B_{u}$ from accessing the MEC network for a predefined time (X-days).

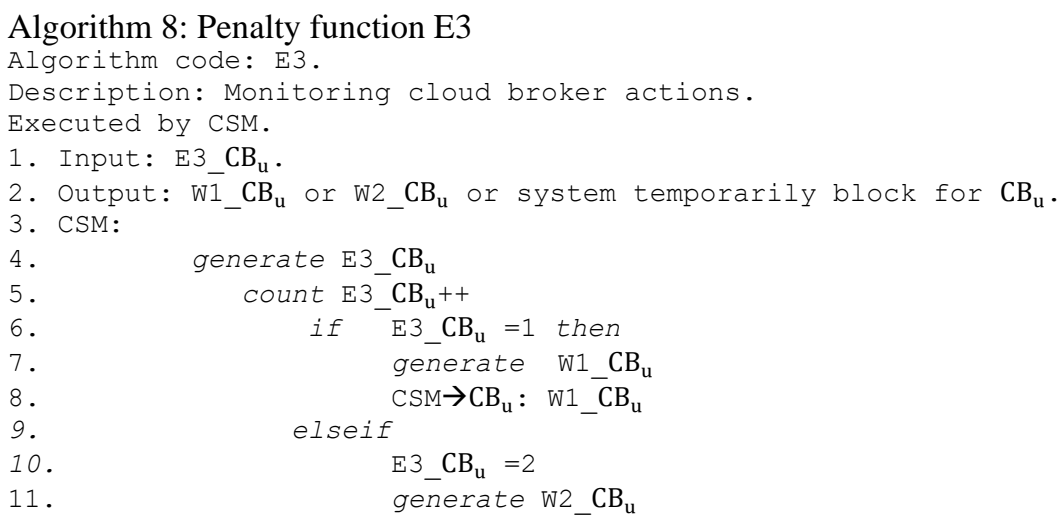




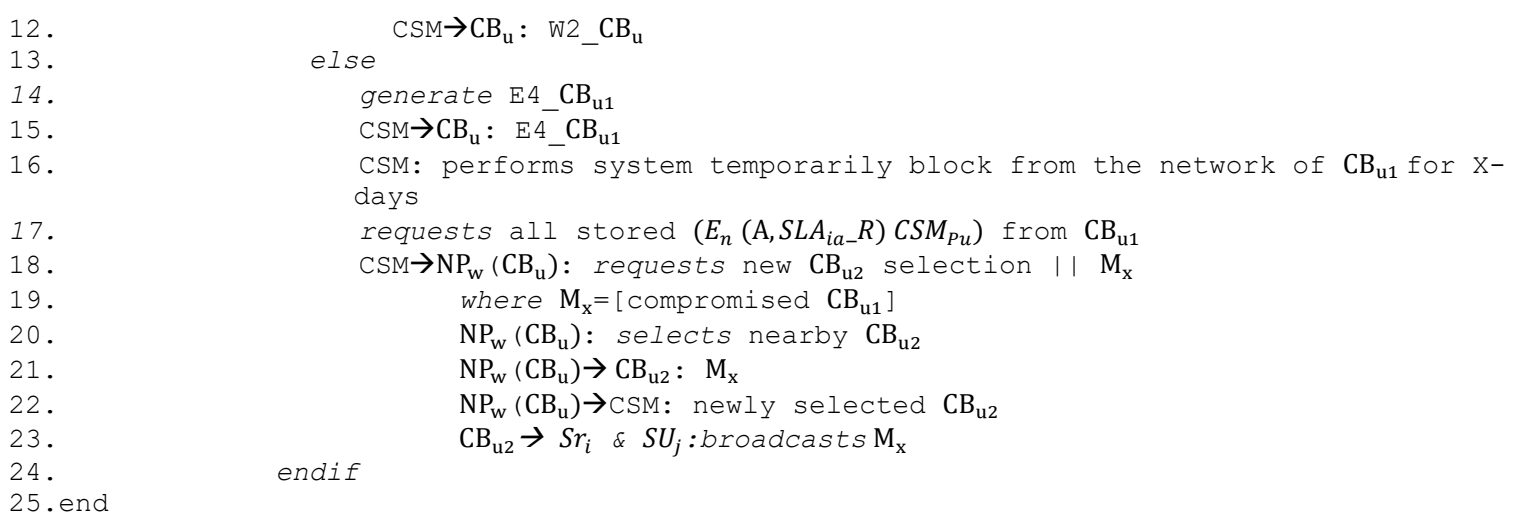

In case of $C B_{u}$ deactivation, CSM requests from the involved network provider to find a subsequent cloud broker and delegates all its functions to the newly selected cloud broker, which continues all the pending jobs. It also broadcasts a warning message $\left(\mathrm{M}_{\mathrm{x}}\right)$ to all surrounding service providers and users, to inform them of the compromised $C B_{u}$. As shown, the penalty functions update the participating entities in case a malicious participant is discovered, where this entity is banned from accessing the MEC network. This optimizes the security of interactions on the MEC network.

\subsubsection{Trust computational algorithms}

Figure 4 shows the processing performance computation steps of $S r_{i}$. Upon completion of function F2 by the cloud broker, the processing performance evaluation of $S r_{i}$, function F4 presented in algorithm 9, is executed by the CSM per computational interval, given that "A $>0$ " ( $S r_{i}$ had received jobs). In case a service provider is working with more than one cloud broker, CSM could authenticate each provider by its unique identity. Function F4 calls two other functions; function F3 (algorithm 10) for processing throughput computation, and function F5 (algorithm 11), that evaluates service provider trust status.

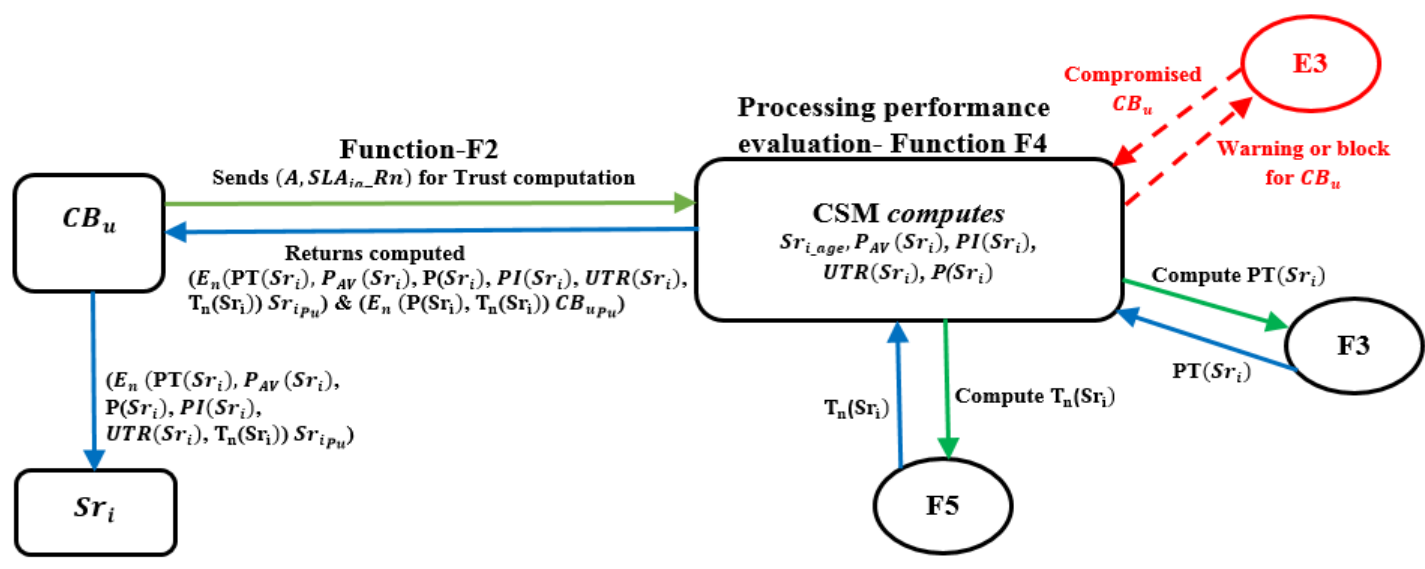

Figure 4. Processing performance computation function-F4

Trust evaluation and all its parameters are evaluated by the CSM for three main reasons: 1- to ensure accurate and fair trust computation for service providers, 2- to guarantee service provider data security and confidentiality, 3- it helps service requesters to reveal trust values remotely prior starting their interactions. $\mathrm{Sr}_{i}$ age is computed to show its processing lifetime in the MEC network. Trust computation begins by computing $P_{E 1 \_} \mathrm{T}, P_{E 2 \_} T, P_{T 1 \_} \mathrm{T}, P_{T 2} \mathrm{~T}$. Each of these parameters indicates the final status per process "a" received by $S r_{i}$. While the computed time difference $T R_{i a} \geq 0$, the average processing success rate $P_{A V}\left(S r_{i}\right)$ is computed. This is because there could be successful processes, in spite that $S r_{i}$ had exceeded the estimated agreed time $S E_{i a}$, but the service user had been patient enough. Therefore, this case is not considered as a fully successful job and could indicate that the predetermined processing capacity is not accurate for this $S r_{i}$. However, this raises the processing throughput value for $\mathrm{Sr}_{i}$ computed by function F3, algorithm 10 . 
While the processing incompliance $P I\left(S r_{i}\right)$ and user termination ratio $U T R\left(S r_{i}\right)$ are computed, in case $\operatorname{UTR}\left(S r_{i}\right)$ exceeds user termination threshold, a warning is generated and sent to the relevant $S r_{i}$ via the $C B_{u}$. This is to alarm $S r_{i}$ of its high user termination ratio. Consequently, the processing performance $\mathrm{P}\left(S r_{i}\right)$ is computed, then function F5 is executed, to get $S r_{i}$ trust status. These results are encrypted by CSM and sent to $S r_{i}$ via its $C B_{u}$. Processing throughput computation, function F3 (algorithm 10), is computed based on the number of successful processes $P_{E 1 \_} T$ executed by $S r_{i}$, (equation (6)). Based on the computed processing performance $\mathrm{P}\left(S r_{i}\right), S r_{i}$ is assigned one of six trust states by function F5, as shown in algorithm 11.

Algorithm 9: Processing performance computation-function F4

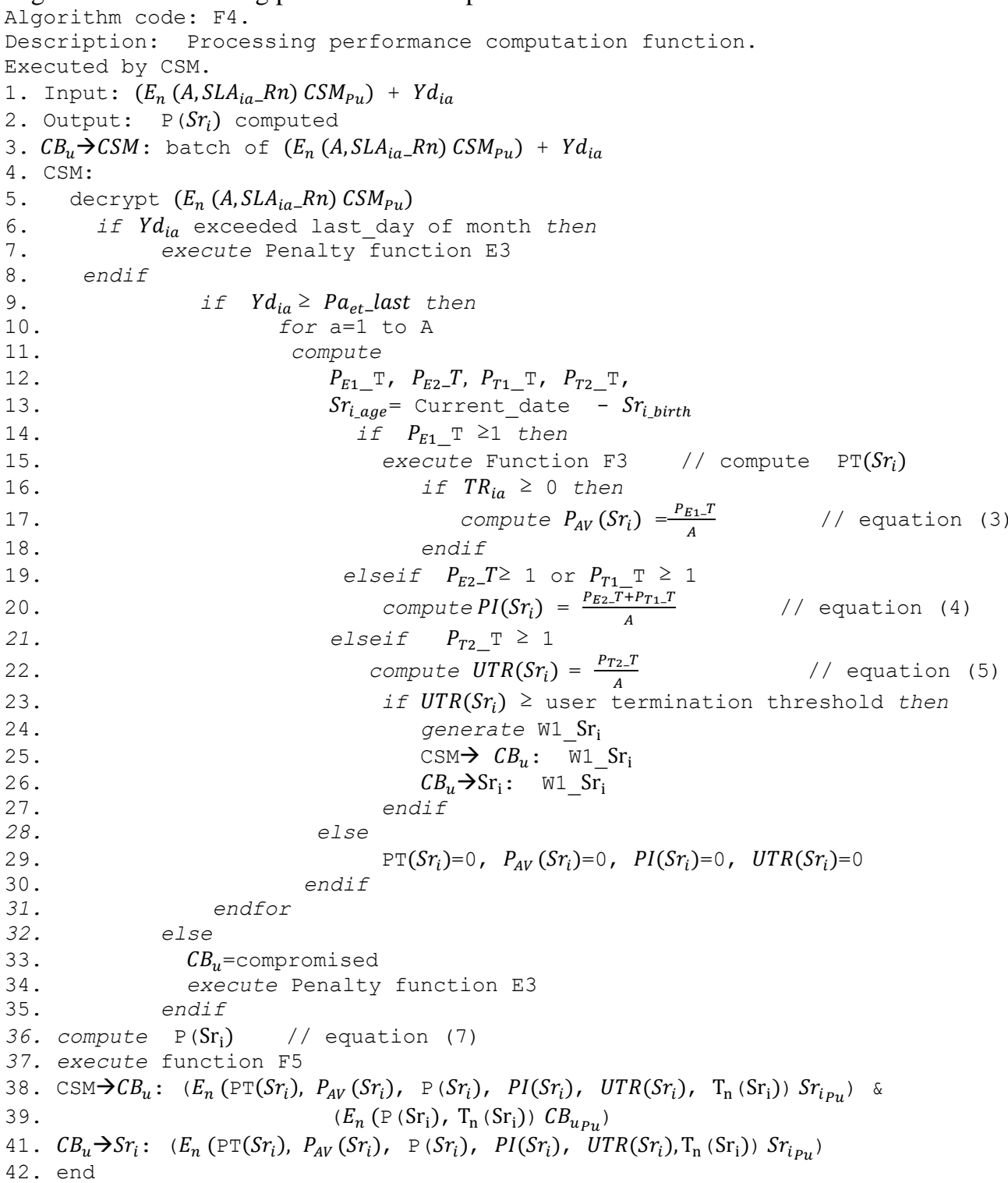

Algorithm 10: Processing throughput computation-function F3

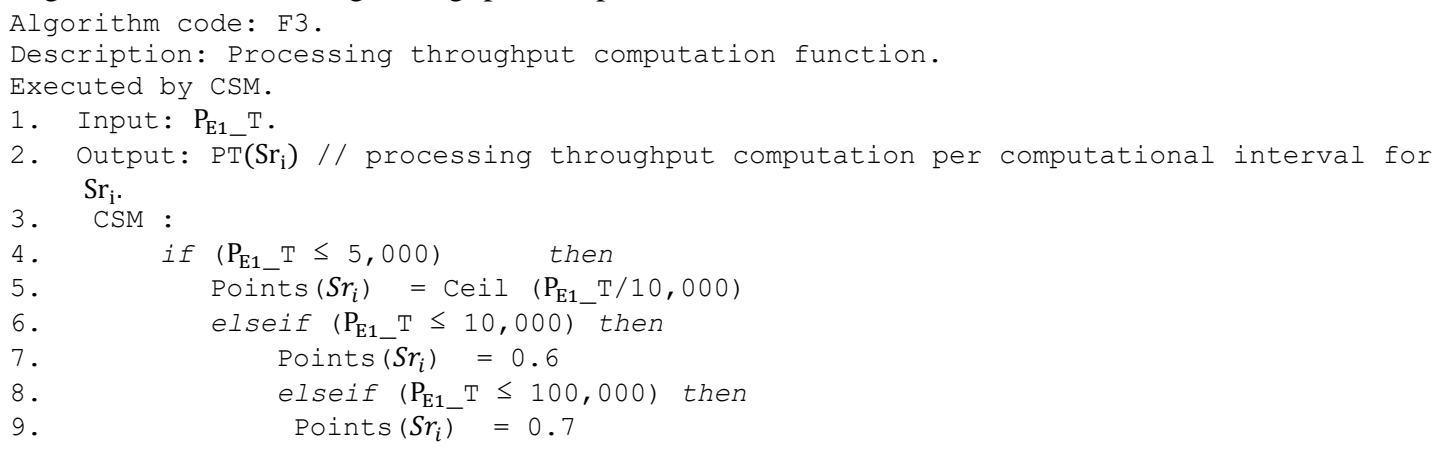




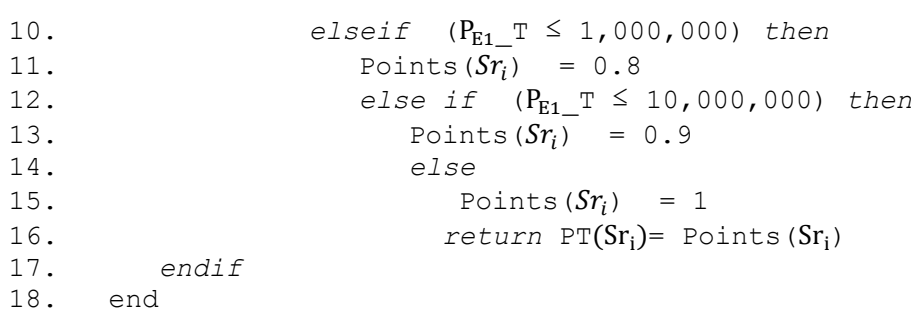

Algorithm 11: Trust status computation algorithm-function F5

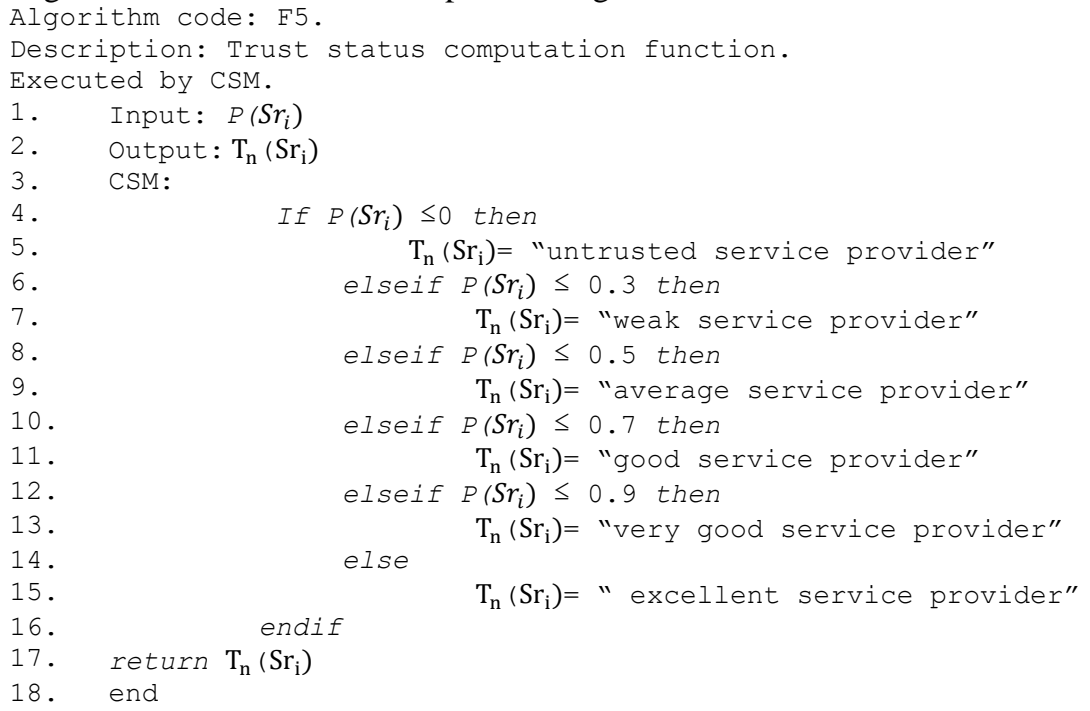

Upon the completion of the above functions, each service provider will be assigned a trust status based on its computed processing performance value. This trust status disseminates service provider's processing performance during its service provisioning in the MEC network.

\section{RESULTS AND DISCUSSION}

Simulation results of the proposed architecture are shown in section 3.1, while the efficiency and effectiveness of proposed scheme are discussed in section 3.2. Section 3.3 presents a comparison between the proposed architecture and some previous protocols.

\subsection{Simulation results}

Simulation of the proposed model was performed using MATLAB program. Simulation setup:

- $\quad$ five different service providers were considered, $S r_{i}=\{1,2,3,4,5\}$.

- initial trust value for each $S r_{i}=$ zero.

- $\quad$ all $S r_{i}$ received the same number of job requests, in one job_type $\{$ Job3 $\}$, from various service users.

- $\quad$ computation intervals $=5$ ( 1 month duration each $)$

- $\quad P_{E 1 \_} \mathrm{T}, P_{E 2 \_} T, P_{T 1 \_} \mathrm{T}, P_{T 2 \_} \mathrm{T}$ values: were assigned to each $S r_{i}$ according to uniform random number generation per month.

- $\quad$ hardware PC configuration = core i7, RAM 6 GB and hard disk 1 Tera.

Trust evaluation was performed over the five months. $S r_{i}$ is evaluated according to its processing performance in its predefined job type. The processing throughput $\mathrm{PT}\left(\mathrm{Sr}_{i}\right)$ results for each service provider per " $m$ " month are shown in Figure 5. While the average processing success rate $P_{A V}\left(S r_{i}\right)$ results are given Figure 6.

Upon measuring the processing throughput and average success rate, the processing performance $\mathrm{P}\left(S r_{i}\right)$ is computed as presented in Figure 7 and the relevant trust status per $S r_{i}$ over the five months, is shown in Table 3. Figure 8 reveals the processing incompliance (failure ratio) $\mathrm{PI}\left(\mathrm{Sr}_{i}\right)$ and Figure 9 shows the user termination ratio per $S r_{i}$. Results show that service providers 1 and 2, trust status had improved gradually by time, because of their improvement in terms of processing throughput and average processing success rate over the five months. With this improve, processing incompliance and user termination ratio, had decreased as shown Figures 8 and 9. Service provider 3 trust status kept varying by time, within a good to 
average range. While service provider 4 maintained its good trust status over the five months, however its processing incompliance acts as a major drawback as illustrated in Figure 8. Service provider 5, kept its excellent processing performance over the five months since its processing incompliance and user termination ratio are very low. Thus, simulation results could identify the processing performance of all five service providers, together with their processing incompliance and user termination ratios, showing their respective trust status.

\subsection{Efficiency and effectiveness of the proposed architecture}

Analysis of the proposed architecture shows that the evaluation time is considerably low, due to the simplicity of the used equations. Processing performance evaluation and trust status results are updated periodically by CSM (fully trusted entity), which increases results credibility. In addition, maintaining a history record decreases service provider trust evaluation time, since it's performed in an accumulative manner. A service provider is registered only once to the MEC network using its unique credentials. This encounters attacks such as fake or malicious service providers, who could deceive users by hiding their bad history.

As the number of service providers increases, the proposed architecture could still distinguish each service provider using its assigned trust status and processing performance value. This validates service providers' computational services trust level and history in the MEC network, which promotes for trusted and secured transactions. A service user is also given a recommendation list of available service providers to choose from, according to user's computational requirements and preferences.

\subsection{Comparison with previous protocols}

Table 4 shows a comparison of the proposed architecture evaluation parameters with previous works. The major limitations/discussion for each one of them.

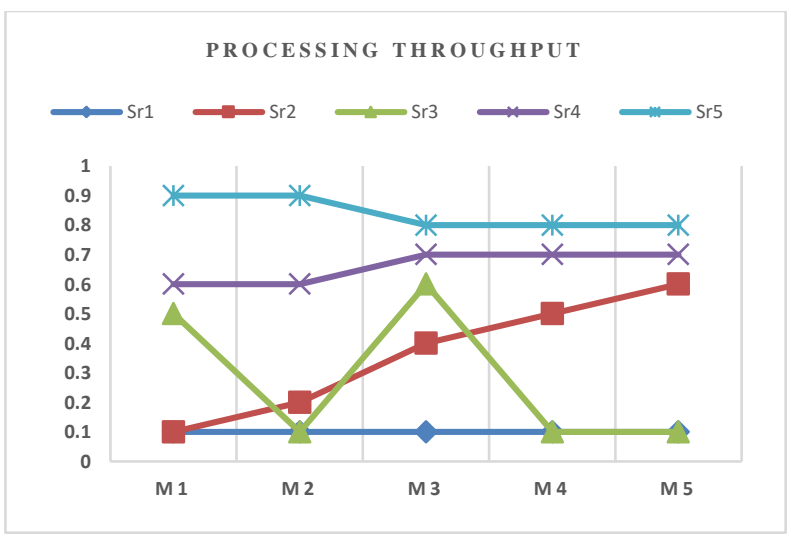

Figure 5. Processing throughput per service provider

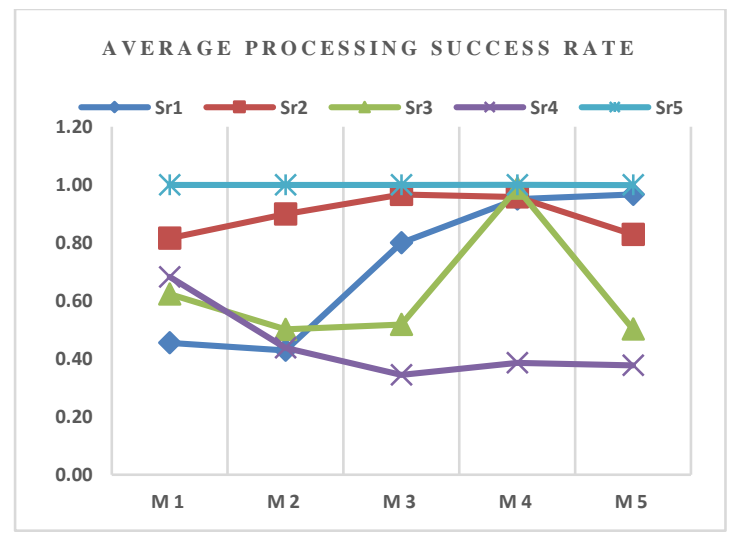

Figure 6. Average processing success rate per service provider

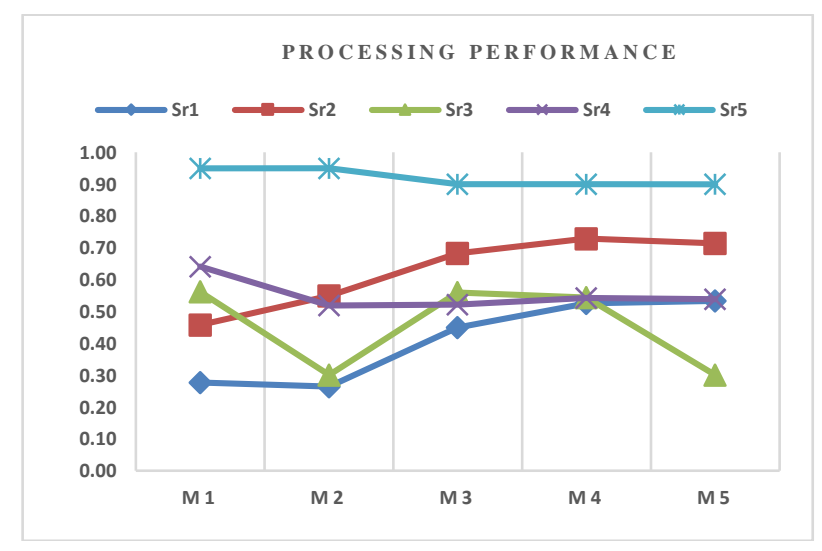

Figure 7. Processing performance per service provider 
Table 3. Trust status per service provider in " $\mathrm{m}$ " months

\begin{tabular}{cccccc}
\hline $\mathrm{Sr}_{i}$ & M1 & M2 & M3 & M4 & M5 \\
\hline $\mathrm{Sr}_{1}$ & Weak & Weak & Average & Good & Good \\
$\mathrm{Sr}_{2}$ & Average & Good & Good & Very good & Very good \\
$\mathrm{Sr}_{3}$ & Good & Average & Good & Good & Average \\
$\mathrm{Sr}_{4}$ & Good & Good & Good & Good & Good \\
$\mathrm{Sr}_{5}$ & Excellent & Excellent & Very good & Very good & Very good \\
\hline
\end{tabular}

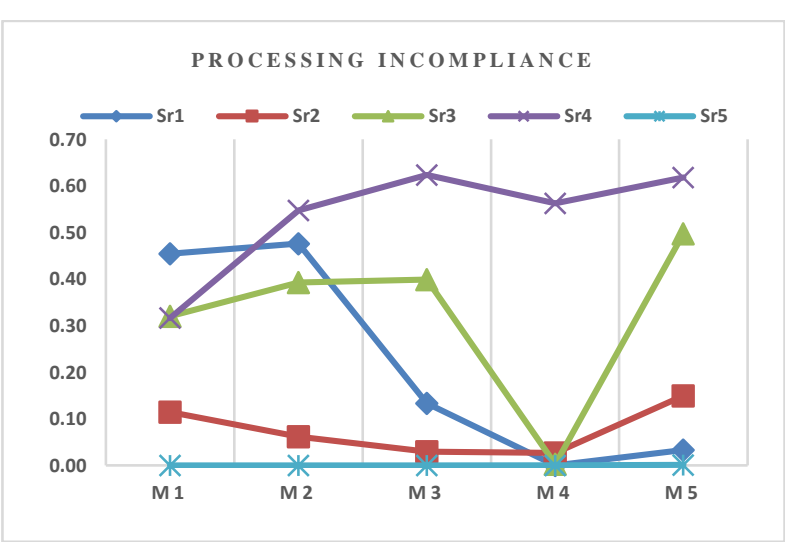

Figure 8. Processing incompliance per service provider

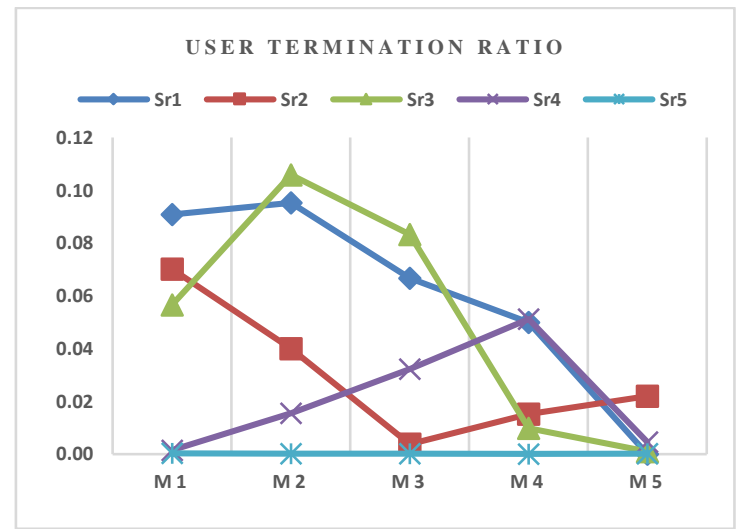

Figure 9. Service providers' user termination ratio

Table 4. Comparison of the proposed architecture and previous work

\begin{tabular}{|c|c|c|c|c|c|}
\hline \multirow{2}{*}{ Protocol } & \multirow{2}{*}{$\begin{array}{l}\text { Service Provider Assessed } \\
\text { Parameters }\end{array}$} & \multirow{2}{*}{$\begin{array}{l}\text { Evaluation } \\
\text { Domain }\end{array}$} & \multicolumn{2}{|c|}{ Trust Update } & \multirow{2}{*}{ Limitations/Discussion } \\
\hline & & & Static & Dynamic & \\
\hline $\begin{array}{l}{[17]} \\
2018\end{array}$ & $\begin{array}{l}\text { Security methods applied in } \\
\text { terms of: i) authentication, ii) } \\
\text { firewall systems; iii) } \\
\text { encryption mechanisms, iv) } \\
\text { intrusion detection }\end{array}$ & $\begin{array}{l}\text { Computation- } \\
\text { based }\end{array}$ & $\sqrt{ }$ & & $\begin{array}{l}\text { Did not consider sudden attacks that could } \\
\text { occur to a node, such as hacking. }\end{array}$ \\
\hline $\begin{array}{l}{[18]} \\
2020\end{array}$ & $\begin{array}{l}\text { Performance in terms of: } \\
\text {-identity authentication } \\
\text {-hardware capabilities } \\
\text {-interactions' behavior }\end{array}$ & $\begin{array}{l}\text { Reputation- } \\
\text { based } \\
\text { (Direct/indirect } \\
\text { trust) }\end{array}$ & & $\sqrt{ }$ & $\begin{array}{l}\text { Depended only on users' opinions. } \\
\text { Collusion attack may occur. }\end{array}$ \\
\hline $\begin{array}{l}{[19]} \\
2018\end{array}$ & $\begin{array}{l}\text { Performance in terms of: } \\
\text {-identity authentication } \\
\text {-capabilities (availability, } \\
\text { response time, throughput, } \\
\text { deployed hardware) } \\
\text {-interactions' behavior }\end{array}$ & $\begin{array}{l}\text { Feedback- } \\
\text { based \& } \\
\text { computation- } \\
\text { based }\end{array}$ & & Partial & $\begin{array}{l}\text { Trust computation is performed by an } \\
\text { unknown entity, which makes trust results } \\
\text { sharing difficult. } \\
\text { Trust computation is complicated and time } \\
\text { consuming. }\end{array}$ \\
\hline $\begin{array}{l}{[20]} \\
2018\end{array}$ & $\begin{array}{l}\text { Analyze traffic flows between } \\
\text { two communicating entities. }\end{array}$ & $\begin{array}{l}\text { Computation- } \\
\text { based }\end{array}$ & & $\sqrt{ }$ & No processing parameters are evaluated. \\
\hline $\begin{array}{l}\text { Proposed } \\
\text { Protocol } \\
2020\end{array}$ & $\begin{array}{l}\text { Processing performance in } \\
\text { terms of: } \\
\text {-processing throughput } \\
\text {-average processing success } \\
\text { rate } \\
\text {-processing incompliance } \\
\text {-user termination ratio. }\end{array}$ & $\begin{array}{l}\text { Computation- } \\
\text { based }\end{array}$ & & $\sqrt{ }$ & $\begin{array}{l}\text { No human interaction involved, which } \\
\text { guarantees results credibility. } \\
\text { History capturing decreases computational } \\
\text { overhead and limits re-register attack. } \\
\text { Dynamic updating of results shows recent } \\
\text { trust status of a service provider in the } \\
\text { MEC network. } \\
\text { Proposed a penalty system to track } \\
\text { malicious entities. }\end{array}$ \\
\hline
\end{tabular}

\section{CONCLUSION AND FUTURE WORK}

Trust was evaluated by computing the processing performance of a service provider, through gaining its average processing success rate and processing throughput. However, processing incompliance and user termination ratio were computed, to accurately determine service providers' performance in the MEC network. The proposed penalty system provided a close monitoring to the participating entities in the MEC network. By capturing the historical trust results, there is no need to evaluate a service provider trust status before the start of each interaction. Thus, gaining accurate and fair trust results with less computation overhead and minimal human interference. 
Simulation results showed that, the higher average processing success rate and throughput, the better processing performance and trust status evaluation gained for a service provider. On the other hand, results illustrated that high processing incompliance or user termination ratio, are reflected in a low processing performance value for a service provider. Thus, maintaining service users' reliability and securing future interactions in the MEC environment. For future work, we plan to evaluate service providers' deployed hardware and software resources. In this context, security measures, scheduling algorithms, fault tolerant protocols deployed by a service provider should be considered during trust evaluation. On the other hand, the number and types of warnings imposed on a service provider, due to performing unauthorized actions should also be considered and analyzed in the future. Given that all acting entities are registered in the network through the network provider, a network provider could act as a cloud broker or even a service provider. On the other hand, a cloud broker could also act as a service provider. However, it will be recommended to review the trust evaluation parameters for these acting entities.

\section{REFERENCES}

[1] S. N. Shirazi, A. Gouglidis, A. Farshad, and D. Hutchison, "The extended cloud: Review and analysis of mobile edge computing and fog from a security and resilience perspective," IEEE Journal on Selected Areas in Communications, vol. 35, no. 11, pp. 2586-2595, Nov. 2017, doi: 10.1109/JSAC.2017.2760478.

[2] H. Li, G. Shou, Y. Hu, and Z. Guo, "Mobile edge computing: progress and challenges," in Proceedings - 2016 4th IEEE International Conference on Mobile Cloud Computing, Services, and Engineering, MobileCloud 2016, 2016, pp. 83-84, doi: 10.1109/MobileCloud.2016.16.

[3] K. Dolui and S. K. Datta, "Comparison of edge computing implementations: fog computing, cloudlet and mobile edge computing," in GIoTS 2017 - Global Internet of Things Summit, Proceedings, 2017, doi: 10.1109/GIOTS.2017.8016213.

[4] B. Liang, "Mobile edge computing," in Key Technologies for 5 G Wireless Systems, V. W. S. Wong, R. Schober, D. W. K. Ng, and L.-C. Wang, Eds. Cambridge University Press, 2017, pp. 76-91.

[5] N. Abbas, Y. Zhang, A. Taherkordi, and T. Skeie, "Mobile edge computing: a Survey," IEEE Internet of Things Journal, vol. 5, no. 1, pp. 450-465, Feb. 2018, doi: 10.1109/JIOT.2017.2750180.

[6] H. El-Sayed et al., "Edge of things: the big picture on the integration of edge, IoT and the cloud in a distributed computing environment," IEEE Access, vol. 6, pp. 1706-1717, 2017, doi: 10.1109/ACCESS.2017.2780087.

[7] S. Wang, X. Zhang, Y. Zhang, L. Wang, J. Yang, and W. Wang, "A survey on mobile edge networks: convergence of computing, caching and communications," IEEE Access, vol. 5, pp. 6757-6779, 2017, doi: 10.1109/ACCESS.2017.2685434.

[8] J. Tan, R. Gandhi, and P. Narasimhan, "Challenges in security and privacy for mobile edge-clouds," Parallel Data Laboratory Carnegie Mellon University Pittsburgh, 2014.

[9] Y. Mao, C. You, J. Zhang, K. Huang, and K. B. Letaief, "A survey on mobile edge computing: the communication perspective," IEEE Communications Surveys and Tutorials, vol. 19, no. 4, pp. 2322-2358, 2017, doi: 10.1109/COMST.2017.2745201.

[10] P. Mach and Z. Becvar, "Mobile edge computing: a survey on architecture and computation offloading," IEEE Communications Surveys and Tutorials, vol. 19, no. 3, pp. 1628-1656, 2017, doi: 10.1109/COMST.2017.2682318.

[11] M. B. Monir, M. H. Abdelaziz, A. A. Abdelhamid, and E. S. M. Ei-Horbaty, "Trust management in cloud computing: a survey," in 2015 IEEE 7th International Conference on Intelligent Computing and Information Systems, ICICIS 2015, 2016, pp. 231-242, doi: 10.1109/IntelCIS.2015.7397227.

[12] A. Ahmed and E. Ahmed, "A survey on mobile edge computing," in Proceedings of the 10th International Conference on Intelligent Systems and Control, ISCO 2016, 2016, doi: 10.1109/ISCO.2016.7727082.

[13] N. Makitalo, A. Ometov, J. Kannisto, S. Andreev, Y. Koucheryavy, and T. Mikkonen, "Safe and secure execution at the network edge: a framework for coordinating cloud, fog, and edge," IEEE Software, pp. 1-1, 2018, doi: 10.1109/MS.2018.110164708.

[14] J. Yuan and X. Li, "A reliable and lightweight trust computing mechanism for IoT edge devices based on multi-source feedback information fusion," IEEE Access, vol. 6, pp. 23626-23638, 2018, doi: 10.1109/ACCESS.2018.2831898.

[15] V. Vassilakis, E. Panaousis, and H. Mouratidis, "Security challenges of small cell as a service in virtualized mobile edge computing environments," in Lecture Notes in Computer Science (including subseries Lecture Notes in Artificial Intelligence and Lecture Notes in Bioinformatics), vol. 9895 LNCS, Springer International Publishing, 2016, pp. 70-84.

[16] F. Pop, C. Dobre, B. C. Mocanu, O. M. Citoteanu, and F. Xhafa, "Trust models for efficient communication in mobile cloud computing and their applications to e-commerce," Enterprise Information Systems, vol. 10, no. 9, pp. 982-1000, Dec. 2016, doi: $10.1080 / 17517575.2015 .1100756$.

[17] F. J. Mora-Gimeno, H. Mora-Mora, D. Marcos-Jorquera, and B. Volckaert, "A secure multi-tier mobile edge computing model for data processing offloading based on degree of trust," Sensors (Switzerland), vol. 18, no. 10, p. 3211, Sep. 2018, doi: $10.3390 / \mathrm{s} 18103211$

[18] X. Deng, J. Liu, L. Wang, and Z. Zhao, "A trust evaluation system based on reputation data in Mobile edge computing network," Peer-to-Peer Networking and Applications, vol. 13, no. 5, pp. 1744-1755, Feb. 2020, doi: 10.1007/s12083-020-00889-3.

[19] X. Ma and X. Li, "Trust evaluation model in edge computing based on integrated trust," in ACM International Conference Proceeding Series, 2018, doi: 10.1145/3302425.3302491.

[20] Y. Ruan, A. Durresi, and S. Uslu, "Trust assessment for internet of things in multi-access edge computing," in Proceedings International Conference on Advanced Information Networking and Applications, AINA, 2018, vol. 2018-May, pp. 1155-1161, doi: 10.1109/AINA.2018.00165

[21] M. H. Ur Rehman, P. P. Jayaraman, S. Ur Rehman Malik, A. Ur Rehman Khan, and M. M. Gaber, "RedEdge: a novel architecture for big data processing in mobile edge computing environments," Journal of Sensor and Actuator Networks, vol. 6, no. 3, p. 17, Aug. 2017, doi: 10.3390/jsan6030017.

[22] S. M. Habib, S. Hauke, S. Ries, and M. Mühlhäuser, "Trust as a facilitator in cloud computing: a survey," Journal of Cloud Computing, vol. 1, no. 1, pp. 1-18, 2012, doi: 10.1186/2192-113X-1-19.

[23] H. Bangui, S. Rakrak, S. Raghay, and B. Buhnova, "Moving to the edge-cloud-of-things: recent advances and future research directions," Electronics (Switzerland), vol. 7, no. 11, p. 309, Nov. 2018, doi: 10.3390/electronics7110309.

[24] J. Huang and D. M. Nicol, "Trust mechanisms for cloud computing," Journal of Cloud Computing, vol. 2, no. 1, p. 9, 2013, doi: $10.1186 / 2192-113 \mathrm{X}-2-9$ 
[25] I. Oteyo, D. P. Mirembe, and M. P. Nampala, "Scaling trust and reputation management in cloud services," International Journal of Applied Science and Technology, vol. 6, pp. 50-57, 2016.

[26] C. Uikey and D. S. Bhilare, "A broker based trust model for cloud computing environment," International Journal of Emerging Technology and Advanced Engineering, vol. 3, no. 11, 2013.

[27] R. Shaikh and M. Sasikumar, "Trust model for measuring security strength of cloud computing service," Procedia Computer Science, vol. 45, no. C, pp. 380-389, 2015, doi: 10.1016/j.procs.2015.03.165.

[28] V. Vassilakis et al., "Security analysis of mobile edge computing in virtualized small cell networks," in IFIP Advances in Information and Communication Technology, vol. 475, Springer International Publishing, 2016, pp. 653-665.

[29] A. Gholami and M. G. Arani, "A trust model based on quality of service in cloud computing environment," International Journal of Database Theory and Application, vol. 8, no. 5, pp. 161-170, Oct. 2015, doi: 10.14257/ijdta.2015.8.5.13.

[30] A. Yousefpour et al., "All one needs to know about fog computing and related edge computing paradigms: a complete survey," Journal of Systems Architecture, vol. 98, pp. 289-330, Sep. 2019, doi: 10.1016/j.sysarc.2019.02.009.

[31] M. B. Monir, T. Abdelkader, and E. S. M. Ei-Horbaty, "Trust evaluation of service level agreement for service providers in mobile edge computing," in Proceedings - 2019 IEEE 9th International Conference on Intelligent Computing and Information Systems, ICICIS 2019, 2019, pp. 362-369, doi: 10.1109/ICICIS46948.2019.9014854.

[32] F. Zohra Filali and B. Yagoubi, "Global trust: a trust model for cloud service selection," International Journal of Computer Network and Information Security, vol. 7, no. 5, pp. 41-50, Apr. 2015, doi: 10.5815/ijcnis.2015.05.06.

[33] F. Meixner and R. Buettner, "Trust as an integral part for success of cloud computing," in ICIW 2012, 2012.

[34] W. Z. Khan, E. Ahmed, S. Hakak, I. Yaqoob, and A. Ahmed, "Edge computing: a survey," Future Generation Computer Systems, vol. 97, pp. 219-235, Aug. 2019, doi: 10.1016/j.future.2019.02.050. 